\title{
Prevalencia y factores asociados a malnutrición en ancianos hospitalizados
}

García Peris P. Prevalencia y factores asociados a malnutrición en ancianos hospitalizados. An Med Interna (Madrid) 2004; 21 : $261-262$.

Se acepta la edad de 65 años como definición de persona mayor. Desde un punto de vista práctico se distinguen tres grupos de edad; de 65 a 74 años hablamos de ancianos jóvenes, de 75 a 84 de ancianos y por encima de 85 años de ancianos viejos.

Cuando hablamos de personas mayores nos estamos refiriendo a un $17 \%$ de la población española, es decir 6.640 millones de personas.

Es importante saber que un 5\% del total vive institucionalizada y un $95 \%$ en su domicilio. Esta distribución es diametralmente opuesta a la que podemos encontrar en el resto de Europa o en EE.UU., donde es frecuente que los ancianos estén en residencias, generalmente por propia voluntad.

En España, de ese 95\% que vive en su domicilio, el $80 \%$ vive en familia y sólo el $20 \%$ vive solo, aunque este porcentaje es mucho mayor que hace unos años y va en aumento.

En el trabajo del Dr. Ramos Martínez (1) sobre "Prevalencia y factores asociados a malnutrición en ancianos hospitalizados", se constatan estos datos demográficos, ya que el grupo estudiado tenía una edad media de 83,0 $\pm 6,4$ años, el $31 \%$ vivía institucionalizado y el $69 \%$ residía en sus domicilios.

La prevalencia de desnutrición en la población anciana que vive en su domicilio varía entre el 3 y el 5\%, sin embargo en los hospitalizados en servicios médicos el porcentaje aumenta al 20-40\%, en los servicios quirúrgicos esta alrededor del $40-60 \%$ y en los institucionalizados puede alcanzar cifras por encima del $60 \%$.

La etiología de la malnutrición es multifactorial. Por un lado están los problemas derivados de la edad avanzada y que afectan de forma importante a todo el funcionamiento del aparato digestivo dando lugar a disfagia, maldigestión, malabsoción, estreñimiento, etc. Por otro lado hay que tener en cuenta problemas socio-económicos (aislamiento social, bajo poder adquisitivo, etc.), problemas psicológicos (trastornos del comportamiento alimentario, depresión) y otros fundamentales como las enfermedades de base y la consiguiente polimedicación con el riesgo de presentar cuadros de interacción fármaco-nutriente, la hospitalización reciente, etc.

De todos es conocido que la desnutrición en cualquier grupo etario está directamente relacionada con un aumento en la mor- bi-mortalidad y empeora de forma determinante la calidad de vida (2), ya que predispone a la aparición de otras patologías, afecta negativamente la capacidad funcional del individuo y aumenta el riesgo de precisar hospitalización y/o institucionalización. Como es lógico, el anciano no escapa a ninguna de estas consecuencias, es más, por la edad es más sensible a ellas.

En el artículo que estamos comentando se constata de forma clara todo lo anteriormente expuesto. Al ingreso en un sevicio de medicina interna se detecta un $57,1 \%$ de ancianos malnutridos y de ellos un $31 \%$ presentaba un deterioro funcional grave.

De acuerdo con la literatura al respecto, en el estudio que comentamos, las enfermedades de base, en este caso EPOC e infecciosas predisponían a la aparición de malnutrición.

Ante esta situación es lógico pensar que es imprescindible realizar una valoración nutricional $(3,4)$ exhaustiva, siempre a esta población.

En la actualidad la valoración del estado nutricional, que es además el primer eslabón del tratamiento nutricional, se subdivide a su vez en dos apartados bien definidos (5): el screening nutricional y la valoración nutricional propiamente dicha.

El objetivo del screening nutricional es identificar a los sujetos malnutridos o que están en riesgo nutricional. Permite identificar personas en las que será necesario realizar una evaluación nutricional más detallada. Por último debe incluir datos objetivos, como la talla, peso, cambios en el peso, enfermedad de base y comorbilidades.

El screening nutricional es muy útil en todas las poblaciones, pero sobre todo en geriatría. Dentro de los tets útiles para realizarlo se incluyen, el Nutrition Screening Initiative (NSI), con el Determine, la Subjetive Global Assessment (VSG) y el Mininutrition Assesment (MNA).

Cuando en el screening nutricional se detecten sujetos en riesgo nutricional, se debe realizar ya una valoración nutricional completa $(6,7)$, que incluye: historia clínica, encuesta dietética, exploración física general antropometría, técnicas de composición corporal más complejas si es posible, determinaciones analíticas, estudios inmunológicos, de capacidad funcional, etc. 
Con respecto a la valoración nutricional conviene hacer algunas precisiones cuando ésta es realizada en personas mayores (8). Así, en la encuesta dietética es importante preguntar quién cocina, si come solo y si toma algún suplemento dietético. Con respecto a la antropometría comentar que el peso siempre debe ser reseñado, así como la pérdida de peso reciente. Si el anciano refiere pérdida de peso, sin causa aparente, es un dato de suma importancia en cuanto al pronóstico y el tratamiento (9). Decir también con referencia al índice de masa corporal (IMC), que el American Commité on Diet and Health ha establecido que IMC inferiores a $24 \mathrm{~kg} / \mathrm{m}^{2}$ y superiores a $29 \mathrm{~kg} / \mathrm{m}^{2}$, no son deseables en individuos de más de 65 años (10). Es decir, parece que los estándares de normalidad en cuanto al IMC, son más altos que para la población menor de 65 años.

En cuanto a los pliegues, comentar que a la hora de interpretarlos no debemos de olvidar que con la edad disminuye la masa magra y aumenta la masa grasa, y que esta se distribuye de forma distinta que en la persona joven, ya que en los ancianos aumenta la grasa intraabdominal y disminuye la subcutánea. Todos estos datos se constatan también, como es lógico, en el estudio del Dr. Ramos Martínez (1).

Con respecto a los parámetros analíticos hacer la reflexión de que en la actualidad a la albúmina se la considera más un indicador de morbi-mortalidad, que nutricional y que con la edad la concentración de albúmina puede disminuir entre un 3 y un $8 \%$ por cada década, después de los 70 años. De todas formas la hipoalbuminemia nunca debería atribuirse a la edad "per se", y siempre es necesario evaluar que causas nutricionales o no nutricionales están implicadas en su aparición. Igualmente descensos significativos en la hemoglobina y en el hematocrito no deben ser atribuidos a la edad. Como la hipoalbuminemia, la anemia en los ancianos debería conducir siempre a descartar una etiología nutricional o no. Téngase en cuenta que la prevalencia de déficit de hierro en las personas mayores es hasta del 44\%. Especial mención hay que hacer también de las vitaminas en esta población, estando indicado realizar siempre que sea posible una valoración de las mismas. De hecho se han descrito deficiencias de vitaminas hidrosolubles, como la vitamina $\mathrm{C}, \mathrm{B}_{6}, \mathrm{~B}_{12} \mathrm{y}$ fólico y de liposolubles, principalmente la vitamina $\mathrm{D}$.

\section{Bibliografía}

1. Ramos Martínez A, Asensio Vegas A, Núñez Palomo S, Millán Santos I. Prevalencia y factores asociados a malnutrición en ancianos hospitalizados. An Med Interna (Madrid) 2004; 21: 263-268.

2. Vetta F, Ranzoni S, Taglieri G, Bollea MR. The impact of malnutrition on the quality of life in the elderly. Clin Nutr 1999; 18: 259-267.

3. Vetta F, Ranzoni S, Palleschi L, et al. Multidimensional approach for nutritional evaluation and restore in the elderly. Clin Nutr 1997; 16 : 269-270.

4. Devons AAJ, Comprehensive geriatric assessment: making the most of the aging years. Curr Opin Clin Nutr Metab Care 2002; 5: 19-24.

5. Guidelines for the use of Parenteral snd Enteral Nutrition in Adult and Pediatric Patients. ASPEN. JPEN 2002; 6: 1 (Sup.)

6. García Peris P, Cuerda Compes C, Camblor Álvarez M, Bretón Lesmes I. Valoración nutricional en el anciano: aspectos practices. Rev Esp
La valoración de la capacidad funcional nunca debe olvidarse en esta población y para ello el indice de Katz o el de Barthel son de gran utilidad, como se demuestra en el estudio a proposito de esta editorial (1). Para la valoración mental es útil el Mini Mental de Folstein.

Un vez conocido el estado nutricional del anciano, si este no fuese el adecuado es necesario hacerle una recomendación en cuanto a su ingesta oral, teniendo en cuenta las recomendaciones de energía, proteínas y micronutrientes acorde a su edad y actividad.

Ahora bien, en muchas ocasiones la alimentación oral no es suficiente para mantener un estado nutricional adecuado. En estos casos en primer lugar se intentará optimizar la dieta oral con consejos en cuanto a número de comidas, consistencia, etc. Si esto no fuese suficiente en la actualidad disponemos de preparados para alimentación oral equilibrados, de diferente textura y sabores, muy útiles en esta población. Cuando estas alternativas fallan debemos recurrir a los suplementos nutricionales y cuando la alimentación oral es insuficiente o no es posible, a la nutrición artificial, enteral o parenteral, según el aparato digestivo sea funcionante o no (11).

Por todo lo anteriormente expuesto resulta muy interesante el artículo del Dr. Ramos Martínez sobre "Prevalencia y factores asociados a malnutrición en ancianos hospitalizados" (1). En primer lugar por incidir como ya hemos dicho en el alto porcentaje de desnutrición en este colectivo y por constatar las variaciones que en el apartado de valoración nutricional hay que tener en cuenta en esta población con respecto a los menores de 65 años, a la hora de interpretar los resultados. Igualmente hay que llamar la atención sobre el alto porcentaje de pacientes que los autores encuentran, con alteración desde el punto de vista funcional, con la negativa repercusión para su calidad de vida.

\section{P. GARCÍA PERIS}

Unidad de Nutrición Clínica. Hospital General Universitario Gregorio Marañón. Madrid
Geritr Gerontol 2000; 35 (S4): 2-8.

7. García Perís P, Serra Rexach JA. Valoración del estado nutricional y valoración geriátrica integral. En: Manual de recomendaciones nutricionales en pacientes geriátricos, Ed. Gómez Candela C y Reus Fernández JM. Ed. Medicos SA. 2004: 43-56.

8. Chernoff R. Normal aging, nutrition, assessment and clinical practice: Clin Nutr Pract 2003; 18: 12-20.

9. Beck AM, Ovensen L. At which body mass index and degree of weight loss should hospitalized elderly patients be considered at nutritional risk? Clin Nutr 1998; 17: 195-8.

10. Ham RS. Indicators of poor nutricional status in olders americans. Am Farm Physician 1992; 45: 219-228.

11. Bozzetti F. Surgery in the elderly: the role of nutritional support. Ciln Nutr 2002; 20: 103-116. 